\title{
Dupilumab Efficacy in Patients Stratified by Baseline Treatment Intensity and Lung Function
}

This article was published in the following Dove Press journal:

Journal of Asthma and Allergy

\author{
Ian D Pavord (D) \\ Salman Siddiqui ${ }^{2}$ \\ Alberto Papi (D) $^{3}$ \\ Jonathan Corren ${ }^{4}$ \\ Lawrence D Sher ${ }^{5}$ \\ Philip Bardin 6 \\ David Langton ${ }^{7}$ \\ Hae-Sim Park (1D ${ }^{8}$ \\ Megan S Rice ${ }^{9}$ \\ Yamo Deniz ${ }^{10}$ \\ Paul Rowe"I \\ Heribert W Staudinger"I \\ Naimish Patel ${ }^{1}$ \\ Marcella Ruddy ${ }^{10}$ \\ Neil MH Graham ${ }^{10}$ \\ Ariel Teper ${ }^{\prime \prime}$

\begin{abstract}
'Respiratory Medicine Unit and Oxford Respiratory National Institute for Health Research Biomedical Research Centre,

University of Oxford, Oxford, UK;

${ }^{2}$ University of Leicester and Leicester

National Institute for Health Research

Biomedical Research Centre (Respiratory

Theme), Leicester, UK; ${ }^{3}$ Department of

Respiratory Medicine, University of Ferrara, Ferrara, Italy; ${ }^{4}$ David Geffen School of

Medicine at UCLA, Los Angeles, CA, USA;

${ }^{5}$ Peninsula Research Associates, Rolling Hills

Estate, CA, USA; ${ }^{6}$ Department of Lung and

Sleep Medicine, Monash University and

Medical Centre, Clayton, Melbourne, VIC,

Australia; ${ }^{7}$ Department of Thoracic

Medicine, Frankston Hospital, Frankston,

Melbourne, VIC, Australia; ${ }^{8}$ Ajou University

Hospital, Suwon, South Korea; 'Sanofi,

Cambridge, MA, USA; ${ }^{10}$ Regeneron

Pharmaceuticals, Inc., Tarrytown, NY, USA;

"Sanofi, Bridgewater, NJ, USA
\end{abstract}

Correspondence: Ian D Pavord NDM Research Building, Nuffield

Department of Medicine, University of

Oxford, Old Road Campus, Roosevelt

Drive, Oxford OX3 7FZ, UK

Tel +44 (0) I865 6I 2897

Email ian.pavord@ndm.ox.ac.uk
Purpose: The Phase 3 LIBERTY ASTHMA QUEST study in patients aged $\geq 12$ years with uncontrolled, moderate-to-severe asthma demonstrated the efficacy and safety of dupilumab $200 \mathrm{mg}$ and $300 \mathrm{mg}$ every 2 weeks (q2w) vs matched placebo in the overall population. This post hoc analysis assessed dupilumab efficacy by disease severity as evidenced by baseline \% predicted forced expiratory volume in 1 second $\left(\mathrm{FEV}_{1}\right)$ and dose of inhaled corticosteroids (ICS).

Patients and Methods: Severe asthma exacerbation rates, change from baseline in $\mathrm{FEV}_{1}$, asthma control, quality of life, and fractional exhaled nitric oxide (FeNO) levels over the 52week treatment period were assessed in patients with elevated type 2 inflammation biomarkers stratified by ICS dose and $\mathrm{FEV}_{1} \%$ predicted at baseline.

Results: In patients with elevated baseline eosinophils, dupilumab $200 \mathrm{mg}$ and $300 \mathrm{mg}$ q2w vs placebo reduced severe exacerbation rates by $50 \%(P=0.06)$ and $67 \%(P=0.001)$, respectively, in those with medium-dose ICS/FEV $\%$ predicted $60-90 \%$, and by $59 \%(P<0.001)$ and $47 \%(P=0.006)$ in those with high-dose $\mathrm{ICS} / \mathrm{FEV}_{1} \%$ predicted $<60 \%$, improved prebronchodilator $\mathrm{FEV}_{1}$ at Week 12 by $0.16 \mathrm{~L}(P=0.005)$ and $0.08 \mathrm{~L}(P=0.13)$, and by $0.20 \mathrm{~L}$ $(P=0.003)$ and $0.21 \mathrm{~L}(P<0.001)$, respectively, in the same subgroups. Dupilumab vs placebo also improved asthma control and quality of life and suppressed FeNO levels in all patient subgroups with similar results observed irrespective of baseline biomarker status or disease severity.

Conclusion: Dupilumab reduced severe exacerbations and improved lung function, asthma control and quality of life in patients with elevated baseline eosinophils irrespective of baseline ICS dose or $\mathrm{FEV}_{1} \%$ predicted.

Keywords: asthma, biomarkers, dupilumab, exacerbation, $\mathrm{FEV}_{1}$

\section{Introduction}

Asthma poses a rising burden for patients, their families, and the community. The severity of asthma can be assessed by examining lung function, as measured by prebronchodilator forced expiratory volume in 1 second $\left(\mathrm{FEV}_{1}\right)$, and the dose of inhaled corticosteroids (ICS) required to maintain disease control. ${ }^{1}$ An assessment of asthma severity enables an appropriate adjustment to treatment to be made in order to reduce the burden to the patient.

Biologic agents for the treatment of moderate-to-severe asthma have demonstrated efficacy in patients with varying degrees of asthma severity as measured by ICS dose alone, but not on the basis of both lung function and ICS dose. ${ }^{2-4}$

Dupilumab, a fully human monoclonal antibody, ${ }^{5,6}$ blocks the shared receptor component for interleukin (IL)-4 and IL-13, ${ }^{7}$ key drivers of type 2 inflammation in multiple diseases, including asthma; ${ }^{8}$ it is approved for patients with type 2 
inflammatory diseases, including atopic dermatitis, asthma, and chronic rhinosinusitis with nasal polyps. ${ }^{9,10}$

The phase 3 LIBERTY ASTHMA QUEST study (NCT02414854) demonstrated the efficacy and safety of dupilumab in patients aged $\geq 12$ years with uncontrolled, moderate-to-severe asthma; ${ }^{11}$ dupilumab significantly reduced the annualized rate of severe exacerbations and improved lung function in the overall population, with greater treatment effects observed in patients with higher levels of type 2 inflammatory biomarkers at baseline.

As QUEST was a large study in which asthma patients were recruited regardless of any phenotype based on baseline biomarkers, the population is diverse and provides the opportunity to assess the efficacy of dupilumab across different patient subgroups. This is of relevance clinically as it enables the identification of patient phenotypes more likely to benefit from dupilumab treatment and indeed other biologics directed against similar targets as they become available.

The aim of this manuscript is to evaluate the efficacy of dupilumab in the patient subgroups of LIBERTY ASTHMA QUEST stratified according to their baseline disease severity, which was defined by their $\mathrm{FEV}_{1} \%$ predicted and ICS dose requirement at baseline.

\section{Methods}

\section{Study Design}

LIBERTY ASTHMA QUEST (NCT02414854) was

a phase 3, randomized, double-blind, placebo-controlled study that assessed the effect of dupilumab in patients with uncontrolled, moderate-to-severe asthma. ${ }^{11}$ Patients aged $\geq 12$ years were randomized in a 2:2:1:1 ratio to add-on subcutaneously administered dupilumab $200 \mathrm{mg}$ (loading dose $400 \mathrm{mg}$ ) or $300 \mathrm{mg}$ (loading dose $600 \mathrm{mg}$ ) every 2 weeks ( 2 w) or matched-volume placebos for 52 weeks. A complete description of the study design is provided in the primary manuscript. ${ }^{11}$

The study was conducted in accordance with the Declaration of Helsinki, the International Conference on Harmonisation Good Clinical Practice guideline, and applicable regulatory requirements. An independent data and safety monitoring committee conducted blinded monitoring of patient safety data. The local institutional review board or ethics committee at each study center oversaw trial conduct and documentation. All patients, or their parents/ guardians, provided written informed consent before participating in the trial. Pediatric patients provided assent according to the Ethics Committee (Institutional Review
Board [IRB]/Independent Ethics Committee)-approved standard practice for pediatric patients at each participating center. The IRB of the study was the Copernicus Group.

\section{Patients}

Complete inclusion and exclusion criteria are provided in the Supplementary Appendix for the primary manuscript. ${ }^{11}$

In order to assess dupilumab efficacy in patients with differing levels of disease severity, patients with moderate-to-severe asthma who had participated in QUEST were stratified according to their requirement of ICS dose (medium or high) and their degree of airflow obstruction $\left(\mathrm{FEV}_{1} \%\right.$ predicted) at baseline, the assumption being that those with poorer airflow obstruction $\left(\mathrm{FEV}_{1} \%\right.$ predicted $<60 \%$ ) and requiring higher doses of ICS to control their disease would have more severe asthma than those on medium-dose ICS and with less severe airflow obstruction. Hence, in this post hoc analysis, four groups of patients were examined: (1) those receiving medium-dose ICS at baseline and with high $\mathrm{FEV}_{1} \%$ predicted (60-80\% for adults, 60-90\% for adolescents), (2) those receiving high-dose ICS at baseline and with low $\mathrm{FEV}_{1} \%$ predicted $(<60 \%)$, (3) those on highdose ICS at baseline and with a $\mathrm{FEV}_{1} \%$ predicted of 60-90\%, and (4) those on medium-dose ICS with a $\mathrm{FEV}_{1} \%$ predicted $<60 \%$ at baseline. Data for groups 1 and 2 are included in the main manuscript (as these represent the two extremes of disease severity), whereas groups 3 and 4 data are included in the supplement, for completeness. As part of the inclusion criteria for QUEST, medium- to high-dose ICS was defined as $\geq 250 \mu \mathrm{g}$ of fluticasone propionate twice daily, or equipotent ICS, to a maximum of $2000 \mu \mathrm{g}$ /day of fluticasone propionate or equivalent. Subgroups of patients who also had elevated baseline levels of the type 2 biomarkers blood eosinophils ( $\geq 150$ cells $/ \mu \mathrm{L})$ or FeNO ( $\geq 25$ parts per billion $[\mathrm{ppb}]$ ) were also examined. These patients are representative of the populations for which dupilumab is indicated in the USA (patients with $\geq 150$ eosinophils $/ \mu \mathrm{L}$ ) and the rest of the world (patients with $\geq 150$ eosinophils/ $\mu \mathrm{L}$ and/or $\mathrm{FeNO} \geq 25 \mathrm{ppb}$ ).

\section{Study Assessments}

The endpoints analyzed in each patient subgroup were annualized severe exacerbation rates, change from baseline in prebronchodilator $\mathrm{FEV}_{1}(\mathrm{~L})$, asthma control (as measured by 5-item Asthma Control Questionnaire [ACQ-5]) and healthrelated quality of life (QoL; as measured by Asthma Quality of 
Life Questionnaire [AQLQ]) scores during the 52-week treatment period. ${ }^{12,13}$ Both the ACQ-5 and AQLQ are patientreported measures; for each the minimum clinically important difference (MCID) is defined as an improvement from baseline of $\geq 0.5$. The effect of treatment on FeNO levels during the 52-week treatment period was also assessed.

\section{Statistical Analysis}

Efficacy analyses were performed in the intent-to-treat (ITT) population, defined as all the patients who underwent randomization; data were analyzed according to the assigned intervention, whether or not it was received. ${ }^{11}$

Annualized rate of severe exacerbations during the 52week treatment period was analyzed using a negative binomial regression model, including the four intervention groups, age, geographic region, baseline eosinophil strata, and number of exacerbations in the previous year as covariates. Patients who discontinued the assigned intervention were encouraged to return to the clinic for all remaining trial visits, and all severe exacerbations up to Week 52 or last contact date (whichever came earlier) were included in the analysis, regardless of whether the patient was receiving an intervention.

Change from baseline in pre-bronchodilator $\mathrm{FEV}_{1}(\mathrm{~L})$, ACQ-5, and AQLQ scores were analyzed using mixedeffects models with repeated measures, including assigned intervention, age, baseline eosinophil strata, visit, intervention-by-visit interaction, region (pooled country), the corresponding baseline value, and baseline-by-visit interaction as covariates. Sex and baseline height were included as additional covariates in the models for $\mathrm{FEV}_{1}$. For patients who discontinued the assigned intervention and remained in the trial, measurements after the intervention was discontinued were included in the analysis.

Differences between dupilumab and matched placebo in the change from baseline in FeNO were analyzed in the exposed population using a rank analysis of covariance (ANCOVA) model, which adjusted for baseline FeNO, age, sex, region (pooled country), and baseline eosinophil strata.

\section{Results}

\section{Baseline Demographic and Clinical Characteristics}

Based on the stratification used in this analysis, 517 patients were classified as receiving medium-dose ICS at baseline with high $\mathrm{FEV}_{1} \%$ predicted; 388 were receiving medium-dose ICS and had low $\mathrm{FEV}_{1} \%$ predicted; 456 were receiving high-dose ICS and had high $\mathrm{FEV}_{1} \%$ predicted and 520 were receiving high-dose ICS and had low $\mathrm{FEV}_{1} \%$ predicted. There were generally few differences in characteristics between dupilumab and matched placebo groups. As would be expected, parameters of lung function, asthma control, and prior exacerbations were worse in the population of patients receiving high-dose ICS at baseline with a lower $\mathrm{FEV}_{1} \%$ predicted (Table 1).

\section{Annualized Rate of Severe Asthma Exacerbations}

Among patients with elevated baseline blood eosinophils, dupilumab reduced severe exacerbation rates vs placebo in both subgroups of patients on medium-dose ICS with high $\mathrm{FEV}_{1} \%$ predicted (by $50 \%$ and $67 \%$ for dupilumab $200 \mathrm{mg}$ and $300 \mathrm{mg}$, respectively; $P=0.06$ and $P=0.001$ ) (Figure 1A) and in those on high-dose ICS with low FEV 1 $\%$ predicted at baseline (by 59\% for dupilumab $200 \mathrm{mg}$, $P<0.001$, and by $47 \%$ for dupilumab $300 \mathrm{mg}, P<0.01$ ) (Figure 1B).

In the subpopulation of patients with elevated blood eosinophils or FeNO at baseline, dupilumab $200 \mathrm{mg}$ and $300 \mathrm{mg}$ vs placebo significantly reduced the severe asthma exacerbation rate by $51 \%(P=0.04)$ and $68 \%(P=0.0004)$, respectively, in patients on medium-dose ICS and with high $\mathrm{FEV}_{1} \%$ predicted (Figure 1C) and by $55 \%$ $(P=0.0009)$ and $44 \%(P=0.0099)$ in patients on high-dose ICS with low $\mathrm{FEV}_{1} \%$ predicted at baseline (Figure 1D).

These improvements in favor of dupilumab were also observed in subgroups of patients stratified by baseline ICS dose and $\mathrm{FEV}_{1 \%}$ predicted only (ie, not stratified by baseline levels of eosinophils or FeNO) (Supplementary Figures 1and 6), with reductions observed for each subgroup.

\section{Pre-Bronchodilator FEV,}

In the patient population with elevated eosinophil count at baseline, dupilumab $200 \mathrm{mg}$ and $300 \mathrm{mg}$ vs placebo improved pre-bronchodilator $\mathrm{FEV}_{1}$ at Week 12 by 0.16 L (95\% confidence interval $[\mathrm{CI}] 0.05,0.27 ; P=0.005$ ) and $0.08 \mathrm{~L}(95 \% \mathrm{CI}-0.02,0.19 ; P=0.131)$, respectively, among patients on medium-dose ICS with high $\mathrm{FEV}_{1} \%$ predicted (Figure 2A) and by $0.20 \mathrm{~L}$ (95\% CI 0.07 , $0.33 ; P=0.003)$ and $0.21 \mathrm{~L}(95 \%$ CI $0.09,0.34$; $P<0.001)$ among patients on high-dose ICS with low $\mathrm{FEV}_{1} \%$ predicted (Figure 2B). Significant improvements 
Table I Baseline Demographic and Clinical Characteristics by Baseline ICS Dose and FEV \% Predicted - ITT Population

\begin{tabular}{|c|c|c|c|c|c|c|c|c|}
\hline \multirow[t]{3}{*}{ Characteristics } & \multicolumn{4}{|c|}{ Medium ICS Dose and FEV $1 \%$ Predicted $60-90 \%$} & \multicolumn{4}{|c|}{ High ICS Dose and FEV $\%$ Predicted $<60 \%$} \\
\hline & \multicolumn{2}{|c|}{$1.14 \mathrm{~mL} / 200 \mathrm{mg} \mathrm{q} 2 \mathrm{w}$} & \multicolumn{2}{|c|}{$2 \mathrm{~mL} / 300 \mathrm{mg} \mathrm{q} 2 \mathrm{w}$} & \multicolumn{2}{|c|}{$1.14 \mathrm{~mL} / 200 \mathrm{mg} \mathrm{q} 2 \mathrm{w}$} & \multicolumn{2}{|c|}{$2 \mathrm{~mL} / 300 \mathrm{mg} \mathrm{q} 2 \mathrm{w}$} \\
\hline & $\begin{array}{l}\text { Placebo } \\
(n=75)\end{array}$ & $\begin{array}{l}\text { Dupilumab } \\
(\mathrm{n}=180)\end{array}$ & $\begin{array}{l}\text { Placebo } \\
(n=91)\end{array}$ & $\begin{array}{l}\text { Dupilumab } \\
(n=|7|)\end{array}$ & $\begin{array}{l}\text { Placebo } \\
(n=80)\end{array}$ & $\begin{array}{l}\text { Dupilumab } \\
(n=176)\end{array}$ & $\begin{array}{l}\text { Placebo } \\
(n=93)\end{array}$ & $\begin{array}{l}\text { Dupilumab } \\
(n=|7|)\end{array}$ \\
\hline Age, mean (SD), years & $42.7(18.3)$ & $44.0(17.1)$ & $43.8(16.7)$ & $43.5(16.7)$ & $49.9(13.7)$ & $51.5(13.1)$ & $50.3(13.8)$ & $50.6(14.1)$ \\
\hline Female, $\mathrm{n}(\%)$ & $43(57.3)$ & $105(58.3)$ & $65(71.4)$ & $105(61.4)$ & $45(56.3)$ & $99(56.3)$ & $56(60.2)$ & $108(63.2)$ \\
\hline Height, mean (SD), cm & $165.4(10.5)$ & $166.9(10.0)$ & $165.2(9.4)$ & $165.3(10.0)$ & $165.4(9.3)$ & $165.5(9.4)$ & $165.9(11.2)$ & $164.1(9.6)$ \\
\hline BMI, mean (SD), $\mathrm{kg} / \mathrm{m}^{2}$ & $26.9(5.8)$ & $28.2(6.6)$ & $28.2(6.9)$ & $28.1(7.2)$ & $31.1(6.8)$ & $29.4(5.8)$ & $30.1(6.5)$ & $29.8(6.6)$ \\
\hline $\begin{array}{l}\text { Pre-bronchodilator } \mathrm{FEV}_{1} \text {, } \\
\text { mean (SD), L }\end{array}$ & $2.2(0.6)$ & $2.2(0.6)$ & $2.1(0.5)$ & $2.2(0.5)$ & $1.4(0.5)$ & $1.4(0.5)$ & $1.4(0.4)$ & $1.4(0.5)$ \\
\hline $\begin{array}{l}\text { Pre-bronchodilator } \mathrm{FEV}_{1} \text {, } \\
\text { mean }(\mathrm{SD}) \text {, } \\
\% \text { predicted }\end{array}$ & $69.5(6.2)$ & $69.3(6.2)$ & $69.8(6.4)$ & $69.4(6.1)$ & $46.9(9.1)$ & $46.6(9.2)$ & $46.5(8.3)$ & $45.5(10.3)$ \\
\hline $\begin{array}{l}\mathrm{FEV} \text {, reversibility, mean }(\mathrm{SD}) \text {, } \\
\%\end{array}$ & $21.9(13.7)$ & $22.7(18.2)$ & $23.2(16.7)$ & $20.5(16.2)$ & $26.3(16.0)$ & $32.2(25.6)$ & $29.7(16.5)$ & $34.5(32.8)$ \\
\hline $\begin{array}{l}\text { Severe asthma exacerbations } \\
\text { in past year, mean (SD), } \mathrm{n}\end{array}$ & $1.7(1.0)$ & $1.8(3.8)$ & $1.9(1.7)$ & $1.8(2.1)$ & $2.4(2.0)$ & $2.1(2.1)$ & $2.7(2.5)$ & $2.2(1.5)$ \\
\hline ACQ-5 score, mean (SD) & $2.6(0.6)$ & $2.5(0.6)$ & $2.6(0.6)$ & $2.6(0.7)$ & $2.9(0.8)$ & $2.9(0.9)$ & $2.9(0.8)$ & $3.0(0.8)$ \\
\hline AQLQ score, mean (SD) & $4.5(1.0)$ & $4.6(1.0)$ & $4.7(0.9)$ & $4.5(1.0)$ & $4.0(1.1)$ & $4.3(1.1)$ & $4.1(1.0)$ & $4.0(1.1)$ \\
\hline $\begin{array}{l}\text { Blood eosinophil count, } \\
\text { median (IQR), cells/ } \mu \mathrm{L}\end{array}$ & $260(130-440)$ & $240(120-440)$ & $210(120-410)$ & $230(130-450)$ & $305(165-600)$ & $250(140-470)$ & $360(180-540)$ & $290(130-500)$ \\
\hline FeNO, median (IQR), ppb & $26(16-56)$ & $25(17-43)$ & $27.50(\mid 8-5 I)$ & $24(13-40)$ & $23(13-38)$ & $24(12-43)$ & $30(15.5-51.5)$ & $25(|5-4|)$ \\
\hline $\begin{array}{l}\text { Serum total IgE, median (IQR), } \\
\mathrm{IU} / \mathrm{mL}\end{array}$ & $\mid 82(8 \mid-493)$ & $153(65-378)$ & $205(53-496)$ & $178(67-546)$ & $225(54-564)$ & $147(49-504)$ & $162(77-319)$ & $192(71-436)$ \\
\hline
\end{tabular}

Abbreviations: ACQ-5, 5-item Asthma Control Questionnaire; AQLQ, Asthma Quality of Life Questionnaire; BMI, body mass index; FeNO, fractional exhaled nitric oxide; $\mathrm{FEV}_{\mathrm{I}}$, forced expiratory volume in I second; ICS, inhaled corticosteroid; ITT, intent-to-treat; IQR, interquartile range; Ppb, parts per billion; q2w, every 2 weeks; SD, standard deviation.

were observed as early as Week 2 for both doses vs placebo and numerical improvements were sustained over the 52-week treatment period for the majority of timepoints in all subgroups.

Also among patients with elevated blood eosinophils or FeNO at baseline, dupilumab vs placebo reduced prebronchodilator $\mathrm{FEV}_{1}$ at Week 12 in subgroups of patients on medium-dose ICS with high $\mathrm{FEV}_{1} \%$ predicted (LS [least squares] mean difference vs placebo: $0.14 \mathrm{~L}$ [95\% CI 0.04, 0.25]; $P=0.008$ with dupilumab $200 \mathrm{mg}$, and $0.09 \mathrm{~L}$ [95\% CI 0, 0.19]; $P=0.06$ with dupilumab $300 \mathrm{mg}$ ) (Figure 2C) and on high-dose ICS with low $\mathrm{FEV}_{1} \%$ predicted $(0.21$ L [95\% CI 0.08, 0.33]; $P=0.001$ with dupilumab $200 \mathrm{mg}$ and $0.21 \mathrm{~L}$ [95\% CI $0.09,0.33$ ]; $P=0.0007$ with dupilumab $300 \mathrm{mg}$ ) (Figure 2D). Improvements vs placebo were observed starting at Week 2, and these were maintained throughout the treatment period.
These improvements vs placebo were also observed in subgroups of patients stratified by baseline ICS dose and $\mathrm{FEV}_{1} \%$ predicted only, with statistically significant improvements observed in each subgroup and for each dupilumab dose at many of the timepoints (see $\underline{\text { Supplementary Figures } 2}$ and 7).

\section{Asthma Control}

Among patients with elevated eosinophil count at baseline, the LS mean change from baseline in ACQ-5 score at Week 24 was -1.45 (standard error [SE] 0.08, difference vs placebo $-0.24[95 \%$ CI $-0.53,0.05] ; P=0.11$ ) with dupilumab $200 \mathrm{mg}$ and -1.31 (SE 0.08 , difference vs placebo $-0.14[95 \% \mathrm{CI}-0.42,0.14] ; P=0.34)$ with dupilumab $300 \mathrm{mg} \mathrm{q} 2 \mathrm{w}$, in patients on medium-dose ICS with high $\mathrm{FEV}_{1} \%$ predicted (Figure $3 \mathrm{~A}$ ), and -1.45 (SE 0.10, difference vs placebo $-0.33[-0.67,0] ; P=0.05)$ and -1.47 
Placebo $1.14 \mathrm{~mL}$ q2w

Dupilumab 200 mg q2w

A

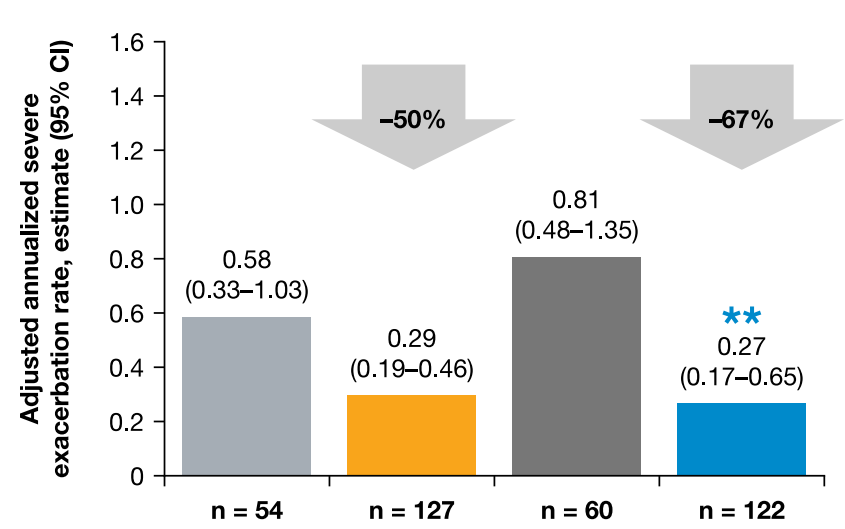

C

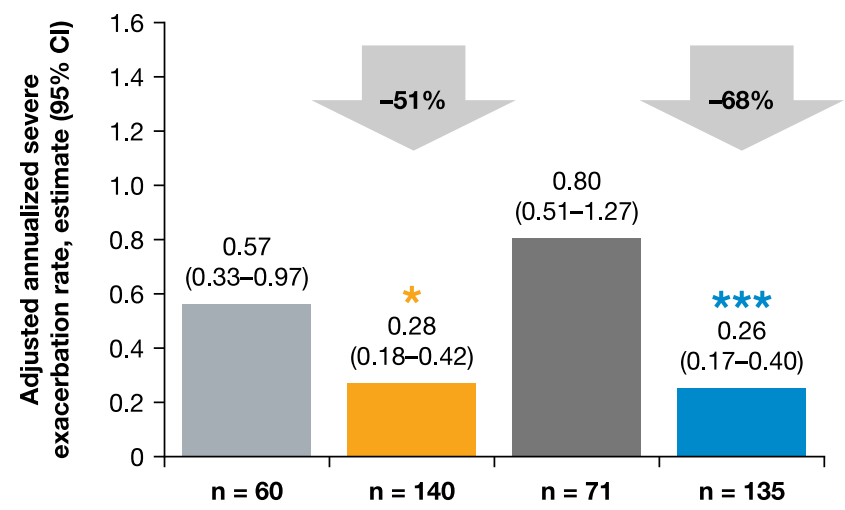

Placebo $2 \mathrm{~mL}$ q2w

Dupilumab 300 mg q2w

B

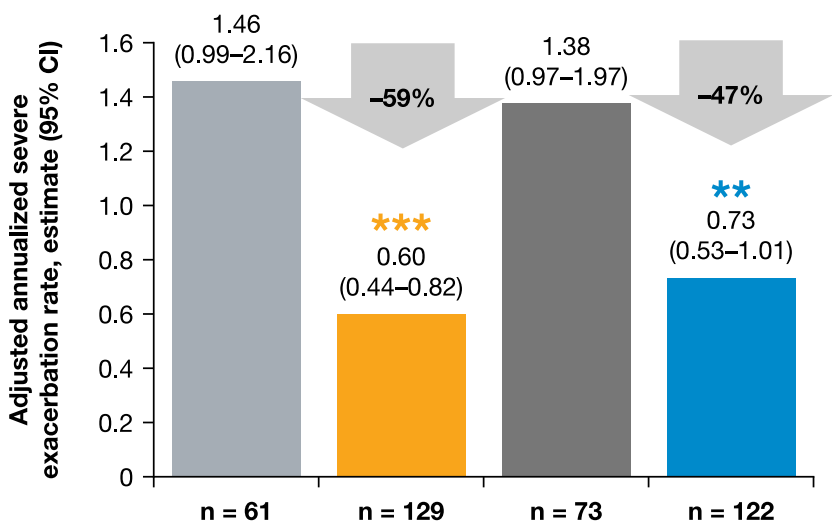

D

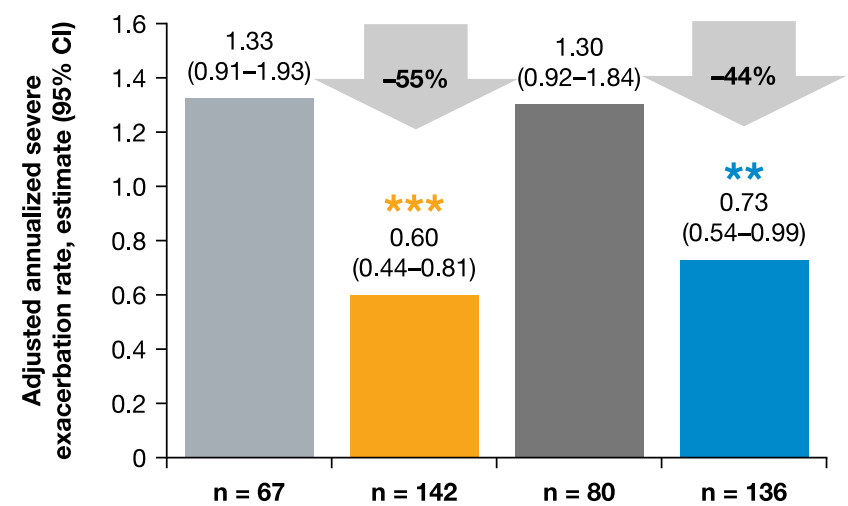

Figure I Annualized rate of severe exacerbations over the 52-week treatment period in patients with (A) medium-dose ICS and $\mathrm{FEV}, \%$ predicted $\geq 60-90 \%$ and $\geq 150$ eosinophils/ $\mu \mathrm{L}$, (B) high-dose ICS and $\mathrm{FEV}_{1} \%$ predicted $<60 \%$ and $\geq 150$ eosinophils/ $\mu \mathrm{L}$, (C) medium-dose ICS and $\mathrm{FEV}, \%$ predicted $\geq 60-90 \%$ and $\geq 150$ eosinophils/ $\mu \mathrm{L}$ or $\geq 25 \mathrm{ppb} F \mathrm{FeNO}$, and (D) high-dose ICS and FEV $\%$ predicted $<60 \%$ and $\geq 150$ eosinophils $/ \mu \mathrm{L}$ or $\geq 25 \mathrm{ppb}$ FeNO at baseline - ITT population. $* * * P<0.00 \mathrm{I}$; $* * P<0.0$ I; $* P<0.05$ vs matched volume placebo.

Abbreviations: $\mathrm{Cl}$, confidence interval; FeNO, fractional exhaled nitric oxide; FEV $\mathrm{F}_{1}$, forced expiratory volume in I second; ICS, inhaled corticosteroids; ITT, intent-to-treat; LS, least squares; ppb, parts per billion; q2w, every 2 weeks.

(SE 0.11 , difference vs placebo $-0.44[95 \%$ CI -0.76 , $-0.12] ; P=0.008$ ), respectively, in patients on high-dose ICS with low $\mathrm{FEV}_{1} \%$ predicted (Figure 3B). Numerical improvements vs placebo were observed starting at Week 2 and were sustained through Week 52 in all subgroups of patients for both dupilumab doses (Figure 3A and B).

In patients with elevated blood eosinophils or FeNO at baseline and on medium-dose ICS with high $\mathrm{FEV}_{1} \%$ predicted, the LS mean change from baseline in ACQ-5 score at Week 24 was -1.44 (SE 0.08, difference vs placebo -0.20 [95\% CI $-0.48,0.07] ; P=0.14$ ) for dupilumab $200 \mathrm{mg}$ and -1.32 (SE 0.08, difference vs placebo -0.16 [95\% CI $-0.42,0.10] ; P=0.23$ ) for dupilumab $300 \mathrm{mg}$ (Figure 3C). Among patients on high-dose ICS with low $\mathrm{FEV}_{1} \%$ predicted, the LS mean change from baseline in
ACQ-5 score was -1.47 (SE 0.10, difference vs placebo -0.29 [95\% CI $-0.61,0.04] ; P=0.08$ ) and -1.43 (SE 0.10, difference vs placebo $-0.37 \quad[95 \%$ CI $-0.68,0.06]$; $P=0.02$ ) for $200 \mathrm{mg}$ and $300 \mathrm{mg}$ doses, respectively (Figure 3D). Numerical improvements vs placebo were observed by Week 2 for both doses and were sustained over the 52-week treatment period (Figure 3C and D).

Across all subgroups, the magnitude of improvements in ACQ-5 observed with add-on dupilumab were clinically meaningful from the first timepoint, exceeding the MCID threshold of 0.5. A placebo effect was observed in the majority of timepoints.

Similar results were observed in subgroups of patients stratified by baseline ICS dose and $\mathrm{FEV}_{1} \%$ predicted only (Supplementary Figures 3 and $\underline{8}$ ). 

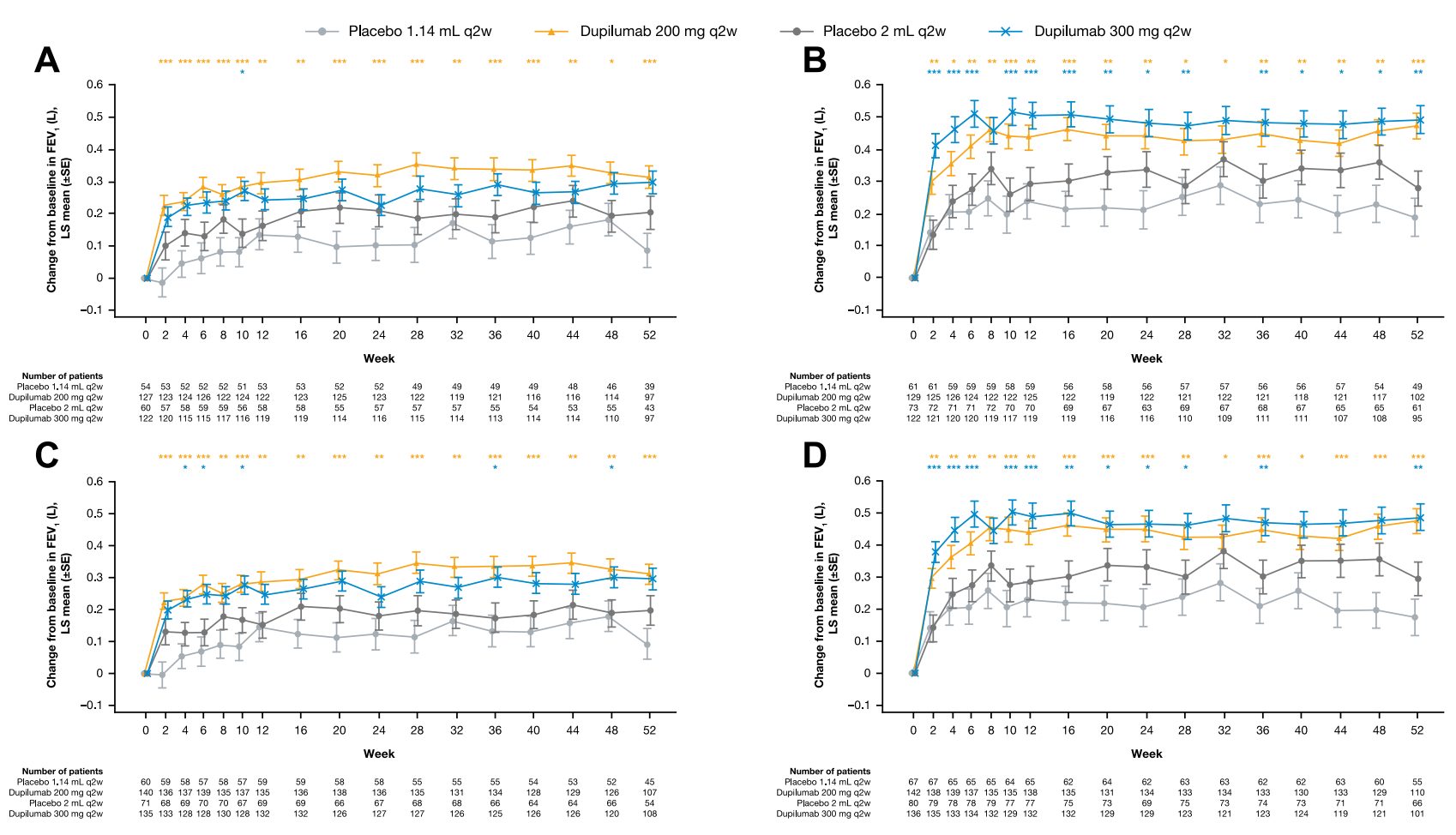

Figure 2 Change from baseline in pre-bronchodilator FEV,$(\mathrm{L})$ over the 52-week treatment period in patients with (A) medium-dose ICS and $\mathrm{FEV}, \%$ predicted $\geq 60-90 \%$

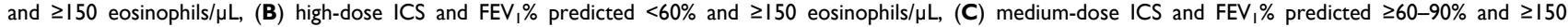

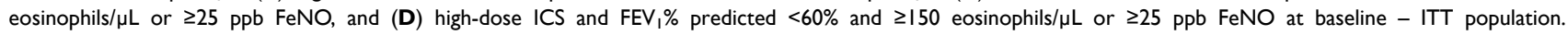
$* * * P<0.00$ I; $* * P<0.01 ; * P<0.05$ vs matched volume placebo.

Abbreviations: FeNO, fractional exhaled nitric oxide; FEV , forced expiratory volume in I second; ICS, inhaled corticosteroids; ITT, intent-to-treat; LS, least squares; Ppb, parts per billion; q2w, every 2 weeks; SE, standard error.

\section{Health-Related QoL}

In patients with elevated blood eosinophils at baseline, among patients on medium-dose ICS with high $\mathrm{FEV}_{1} \%$ predicted, the LS mean change from baseline in AQLQ score at Week 24 was 1.07 (SE 0.09) for dupilumab $200 \mathrm{mg}$ (LS mean difference vs placebo 0.18 [95\% CI $-0.13,0.49] ; P=0.252$ ) and 1.01 (SE 0.09) for dupilumab $300 \mathrm{mg}$ (LS mean difference vs placebo 0.10 [95\% CI $-0.20,0.40] ; P=0.498$ ) (Figure $4 \mathrm{~A}$ ) and among patients on high-dose ICS with low $\mathrm{FEV}_{1} \%$ predicted was 1.11 (SE 0.10) for dupilumab $200 \mathrm{mg}$ (LS mean difference vs placebo $0.13 \quad[95 \%$ CI $-0.19,0.45]$; $P=0.428$ ) and 1.22 (SE 0.10) for dupilumab $300 \mathrm{mg}$ (LS mean difference vs placebo $0.30\left[\begin{array}{llll}95 \% & \text { CI } & 0 \text {, }\end{array}\right.$ 0.60]; $P=0.0496$ ) (Figure 4B). Numerical improvements vs placebo were observed over the 52-week treatment period with these reaching statistical significance at some timepoints during the 52-week-treatment period (Figure 4A and B).

In the subgroup of patients with elevated blood eosinophils or FeNO $\geq 25 \mathrm{ppb}$ at baseline, LS mean change from baseline in AQLQ score at Week 24 was 1.04 (SE 0.08) (LS mean difference vs placebo 0.14 [95\% CI $-0.14,0.43] ; P=0.3281$ ) with dupilumab $200 \mathrm{mg}$ and 1.00 (SE 0.08) with dupilumab $300 \mathrm{mg}$ (LS mean difference vs placebo 0.06 [95\% CI $-0.21,0.33]$; $P=0.6871)$ in patients on medium-dose ICS with high $\mathrm{FEV}_{1} \%$ predicted (Figure 4C) and was 1.14 (SE 0.09; LS mean difference vs placebo 0.13 [95\% CI -0.18 , 0.43]; $P=0.4145$ ) and 1.21 (SE 0.09; LS mean difference vs placebo $0.24[95 \%$ CI $-0.04,0.52] ; P=0.0986$ ), respectively, in patients on high-dose ICS with low $\mathrm{FEV}_{1} \%$ predicted (Figure 4D). Numerical improvements vs placebo, some significant, were observed over the 52week treatment period (Figure 4C and D).

At all timepoints, the improvements in AQLQ observed with add-on dupilumab were clinically significant, exceeding the MCID of 0.5, although these benefits were not always statistically significant vs placebo treatment. A placebo effect was also observed.

Numerical, but non-significant improvements in AQLQ vs placebo were observed with dupilumab in subgroups of 

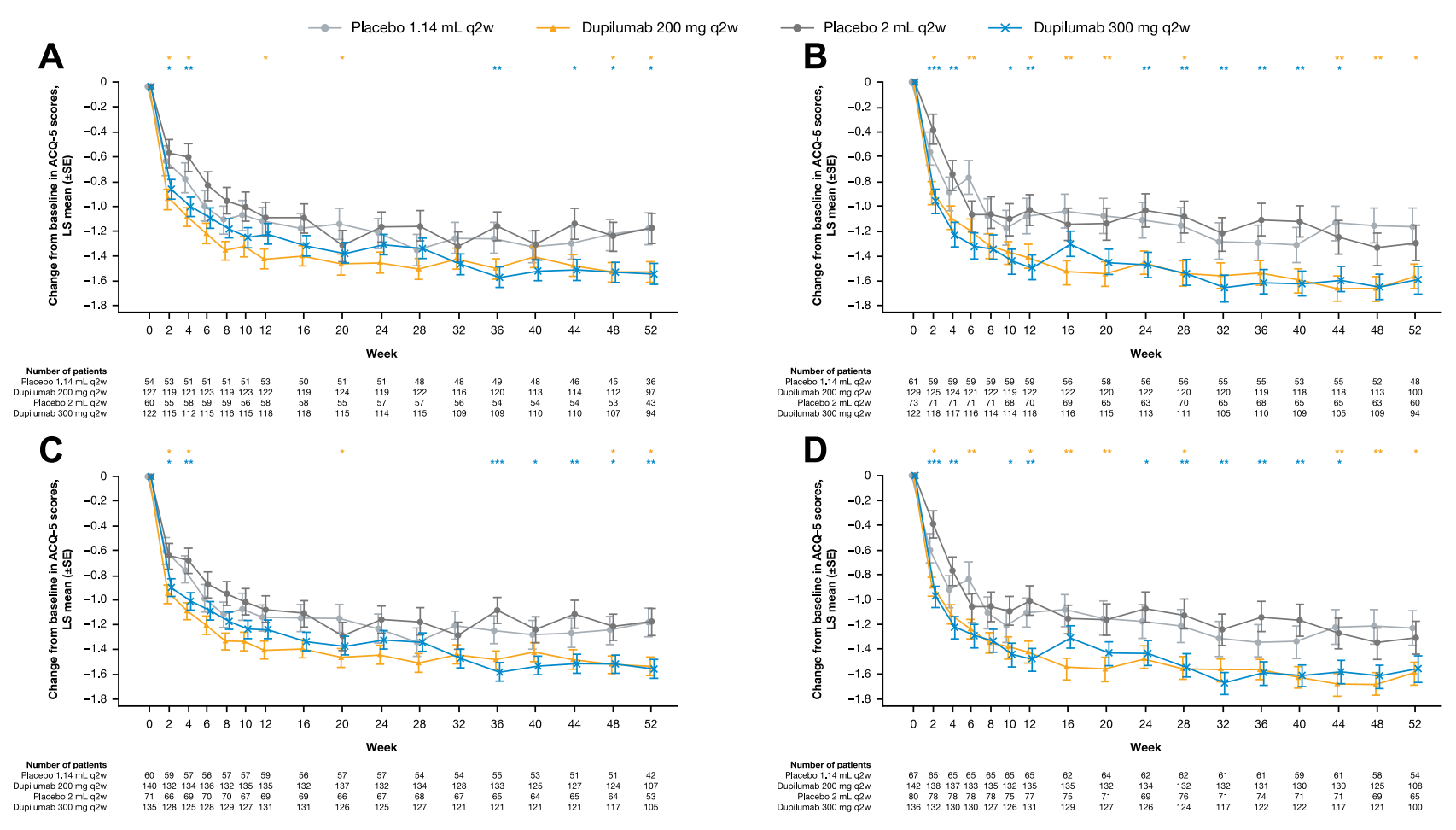

Figure 3 Change from baseline in ACQ-5 score over the 52-week treatment period in patients with (A) medium-dose ICS and FEV,$\%$ predicted $\geq 60-90 \%$ and $\geq 150$ eosinophils/ $\mu \mathrm{L}$, (B) high-dose ICS and $\mathrm{FEV}_{1} \%$ predicted $<60 \%$ and $\geq 150$ eosinophils/ $\mu \mathrm{L}$, (C) medium-dose ICS and FEV $\%$ predicted $\geq 60-90 \%$ and $\geq 150$ eosinophils/ $\mu \mathrm{L}$ or $\geq 25 \mathrm{ppb} F \mathrm{NeNO}$, and (D) high-dose ICS and FEV $\%$ predicted $<60 \%$ and $\geq 150$ eosinophils $/ \mu \mathrm{L}$ or $\geq 25 \mathrm{ppb}$ FeNO at baseline - ITT population. $* * * P<0.00 \mathrm{I}$; $* * P<0.0 \mathrm{I}$; $* P<0.05$ vs matched volume placebo.

Abbreviations: ACQ-5, 5-item Asthma Control Questionnaire; FeNO, fractional exhaled nitric oxide; FEV 1 , forced expiratory volume in I second; ICS, inhaled corticosteroids; ITT, intent-to-treat; LS, least squares; ppb, parts per billion; q2w, every 2 weeks; SE, standard error.

patients stratified by baseline ICS dose and $\mathrm{FEV}_{1} \%$ predicted only (Supplementary Figures 4 and 9 ).

\section{$\mathrm{FeNO}$}

Among patients with elevated blood eosinophils at baseline, dupilumab $200 \mathrm{mg}$ and $300 \mathrm{mg}$ q2w treatment significantly reduced FeNO levels vs placebo in patients on mediumdose ICS with high $\mathrm{FEV}_{1} \%$ predicted as well as in patients on high-dose ICS with low $\mathrm{FEV}_{1} \%$ predicted (all $P<0.01$ at all timepoints) (Figure 5A and B). At Week 52, the median FeNO level was $18.0 \mathrm{ppb}$ and $15.0 \mathrm{ppb}$ with dupilumab $200 \mathrm{mg}$ and $300 \mathrm{mg}$ (33.0 and $35.0 \mathrm{ppb}$ for matched placebos), respectively, in patients on medium-dose ICS with high $\mathrm{FEV}_{1} \%$ predicted and $15.0 \mathrm{ppb}$ and $16.0 \mathrm{ppb}$ (20.0 and $29.5 \mathrm{ppb}$ for matched placebos), respectively, in patients on high-dose ICS with low $\mathrm{FEV}_{1} \%$ predicted. Suppression of FeNO to within normal ranges occurred rapidly, near maximal suppression of FeNO was observed by the first assessment at Week 2 and it was maintained throughout the 52-week treatment period.

Comparable suppression was observed in the subgroups of patients who had elevated baseline levels of blood eosinophils or FeNO (Figure 5C and D), and in the subgroups of patients stratified by baseline ICS dose and $\mathrm{FEV}_{1} \%$ predicted only (Supplementary Figures 5 and 10 ).

\section{Discussion}

In this post hoc analysis of QUEST, dupilumab demonstrated significant efficacy in patients with uncontrolled, moderate-to-severe asthma, regardless of their baseline disease severity defined by baseline ICS requirement or $\mathrm{FEV}_{1} \%$ predicted. Furthermore, dupilumab was also effective in subpopulations of patients with elevated baseline levels of the type 2 biomarkers blood eosinophils and FeNO, on medium- or high-dose ICS, and with high or low $\mathrm{FEV}_{1} \%$ predicted at study entry.

In this analysis, dupilumab significantly reduced the annualized severe exacerbation rate and improved lung function $\left(\mathrm{FEV}_{1}\right)$ in patients with elevated levels of the type 2 biomarkers blood eosinophils or FeNO at baseline, with treatment effects observed in the subgroups of patients on medium-dose ICS and high $\mathrm{FEV}_{1} \%$ predicted, and high-dose ICS and low $\mathrm{FEV}_{1} \%$ predicted (the 2 extremes of the severity spectrum). As has been reported 


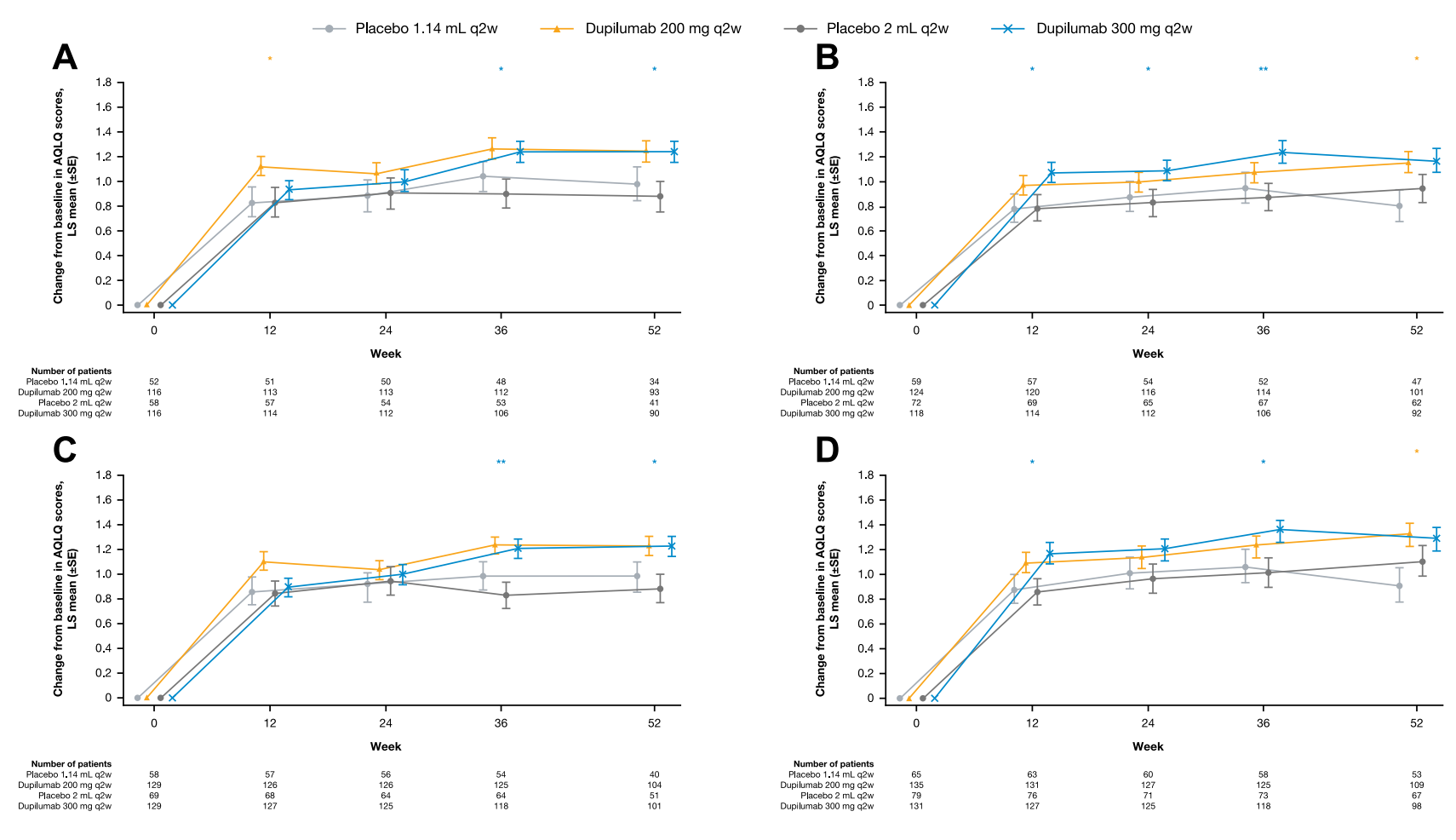

Figure 4 Change from baseline in AQLQ score over 52 weeks in patients with (A) medium-dose ICS and FEV $1 \%$ predicted $\geq 60-90 \%$ and $\geq 150$ eosinophils/ $\mu \mathrm{L}$, (B) high-dose ICS and $\mathrm{FEV}_{1} \%$ predicted $<60 \%$ and $\geq 150$ eosinophils/ $\mu \mathrm{L}$, (C) medium-dose ICS and FEV $1 \%$ predicted $\geq 60-90 \%$ and $\geq 150$ eosinophils/ $\mu \mathrm{L}$ or $\geq 25$ ppb FeNO, and (D) highdose ICS and $\mathrm{FEV}, \%$ predicted $<60 \%$ and $\geq 150$ eosinophils $/ \mu \mathrm{L}$ or $\geq 25 \mathrm{ppb}$ FeNO at baseline - ITT population. $* * P<0.01$; $* P<0.05$ vs matched volume placebo.

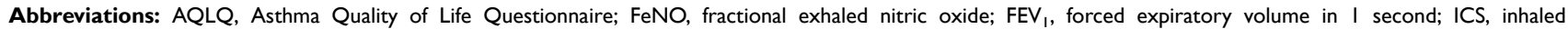
corticosteroids; ITT, intent-to-treat; LS, least squares; ppb, parts per billion; q2w, every 2 weeks; SE, standard error.

previously, improvements in $\mathrm{FEV}_{1}$ were observed as early as 2 weeks after treatment onset and were maintained throughout the 52-week treatment period in all subpopulations examined, with statistically significant improvements observed at many of the timepoints. Similar findings were observed in the subgroups of patients on medium-dose ICS with low $\mathrm{FEV}_{1} \%$ predicted and on high-dose ICS with high $\mathrm{FEV}_{1} \%$ predicted.

These results suggest that not only is dupilumab efficacious in patients with elevated blood eosinophils or FeNO levels, but it is also efficacious irrespective of baseline ICS requirement or $\mathrm{FEV}_{1} \%$ predicted. This is an important finding as other biologic agents have shown efficacy in patients with either moderate or severe asthma, but not in both. Of other asthma biologics available, early studies with the anti-IL-5 antibodies mepolizumab and reslizumab did not demonstrate significant clinical benefit vs placebo in patients with moderately severe asthma with persistent symptoms despite receiving $\mathrm{ICS}^{2}$ or significant effect on lung function $\left(\mathrm{FEV}_{1}\right)^{3}$ in patients with severe asthma also on ICS controller medication. However, these early studies did not select patients according to type 2 biomarkers, and no examination of the stratified data has been published. Nevertheless, two later studies with reslizumab reported significant improvements in exacerbation rates and numerical improvements in lung function in patients with moderate-to-severe asthma with an eosinophilic phenotype. ${ }^{14}$ The utility of biomarkers in predicting clinical benefit has also been described for the anti-IgE antibody omalizumab in allergic asthma, where greater treatment effects were observed in patient subgroups defined by elevated levels of FeNO, eosinophils, and serum periostin. ${ }^{4}$ Overall, this post hoc analysis has confirmed that the efficacy of dupilumab $\mathrm{q} 2 \mathrm{w}$ demonstrated in the overall population of the LIBERTY ASTHMA QUEST study is also evident in subgroups of patients with moderate and severe disease at study entry, with many of these benefits sustained over time. The greater treatment effects seen in patients who had higher baseline blood eosinophil counts or FeNO concentrations - recognized biomarkers of type 2 inflammation - were consistent with previous dupilumab asthma studies. ${ }^{11,15,16}$ Furthermore, and consistent with its biologic action, dupilumab treatment vs placebo resulted in sustained suppression of FeNO throughout the treatment period, with near-maximal suppression observed by the first assessment at Week 2 . 

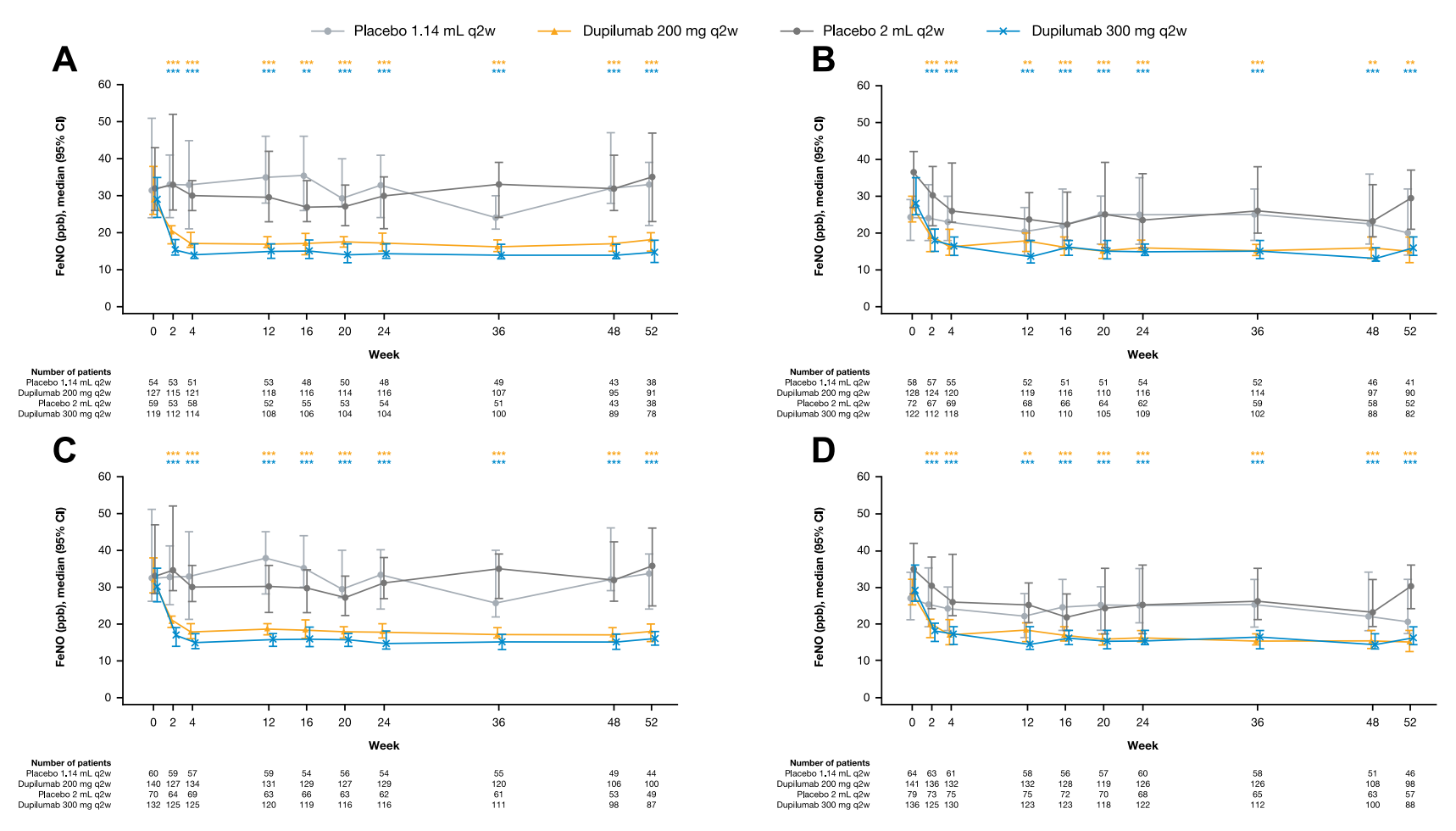

Figure 5 Median FeNO (ppb) during the 52-week treatment period in patients with (A) medium-dose ICS and FEV $\%$ predicted $\geq 60-90 \%$ and $\geq I 50$ eosinophils/ $\mu \mathrm{L}$, (B)

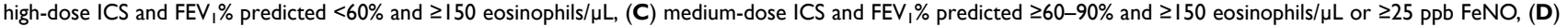
high-dose ICS and $\mathrm{FEV}, \%$ predicted $<60 \%$ and $\geq 150$ eosinophils/ $\mu \mathrm{L}$ or $\geq 25 \mathrm{ppb}$ FeNO at baseline - exposed population. $* * * P<0.00 \mathrm{I}$; $* * P<0.0 \mathrm{I}$ vs matched volume placebo ( $P$-values based on change from baseline vs placebo).

Abbreviations: $\mathrm{Cl}$, confidence interval; FeNO, fractional exhaled nitric oxide; $\mathrm{FEV}_{1}$, forced expiratory volume in I second; ICS, inhaled corticosteroids; Ppb, parts per billion; q2w, every 2 weeks; SE, standard error.

Patients enrolled in QUEST presented uncontrolled asthma with high baseline FeNO levels, which is generally correlated with poor outcomes. Our data highlight dupilumab's efficacy in this difficult-to-treat population, as it reduces FeNO levels as early as Week 2, which were sustained throughout the treatment period.

That dupilumab appears to be able to improve lung function in moderate-to-severe asthma patients with elevated biomarkers of type 2 inflammation may be explained by the fact that it targets and inhibits both IL-4 and IL-13 signaling, which are important cytokines that contribute to type 2 inflammation in asthma patients. ${ }^{8,17}$ IL-4 plays a central role in type 2 $\mathrm{T}$ helper cell differentiation, inducing the production of type 2-associated cytokines and chemokines (such as IL-5, IL-9, IL13, TARC, and eotaxins), as well as promoting isotype class switching of B cells to produce IgE and recruit eosinophils to areas of inflammation. ${ }^{17,18}$ IL-13 on the other hand, mediates goblet cell hyperplasia and the concomitant hypersecretion of mucus, and enhances smooth muscle contractility and airway hyper responsiveness. ${ }^{18}$

With regard to asthma control and health-related QoL, improvements in ACQ-5 and AQLQ scores were not as marked in patients stratified by ICS use and $\mathrm{FEV}_{1} \%$ predicted at baseline. However, differences vs placebo were of comparable magnitude to that observed in the overall ITT population. ${ }^{11}$ The ACQ-5 and AQLQ are traditional measures of asthma severity; however, unlike biomarkers, they have no predictive potential as to the likelihood of a response. Conversely, biomarker analysis is a valuable approach, which facilitates the pre-treatment stratification of patients into those more likely to respond thereby enabling the best use of new targeted treatments.

As previously reported, dupilumab was generally well tolerated in the overall safety population. ${ }^{11}$ The post hoc nature of the present analysis represents a limitation, as the study was not powered to specifically investigate differences in subpopulations stratified by baseline ICS dose and $\mathrm{FEV}_{1} \%$ predicted.

\section{Conclusion}

In conclusion, this post hoc analysis has demonstrated that dupilumab is efficacious in patients with moderate asthma and in those with severe asthma as defined by baseline ICS dose requirement and $\mathrm{FEV}_{1} \%$ predicted. These are 
important findings that build on previous knowledge on the efficacy of dupilumab as they provide information on the patients likely to benefit from treatment and will be of use in informing treatment decisions.

Furthermore, we have also confirmed that the benefit of dupilumab demonstrated in the overall population of the LIBERTY ASTHMA QUEST study is also evident in subgroups of patients with elevated biomarkers of type 2 asthma, stratified by baseline ICS dose and $\mathrm{FEV}_{1} \%$ predicted, with efficacy observed early and sustained over time. As such, the findings suggest that dupilumab should be considered as an add-on maintenance treatment for uncontrolled, moderate-to-severe asthma in patients with elevated blood eosinophils or FeNO, on medium-dose ICS and $\mathrm{FEV}_{1} \%$ predicted $\geq 60-90 \%$, as well as patients on high-dose ICS and $\mathrm{FEV}_{1} \%$ predicted $<60 \%$.

\section{Abbreviations}

ACQ-5, 5-item Asthma Control Questionnaire; AQLQ, Asthma Quality of Life Questionnaire; CI, confidence interval; FeNO, fractional exhaled nitric oxide; $\mathrm{FEV}_{1}$, forced expiratory volume in 1 second; ICS, inhaled corticosteroids; IL, interleukin; ITT, intent-to-treat; LS, least squares; ppb, parts per billion; q2w, every 2 weeks; QoL, quality of life; SC, subcutaneous; SE, standard error; TARC, thymus and activation-regulated chemokine.

\section{Acknowledgments}

Regeneron: Nora Crikelair, Sanofi: Dianne Barry. The Liberty Asthma QUEST trial was sponsored by Sanofi and Regeneron Pharmaceuticals Inc. Medical writing/editorial assistance in the development of this manuscript was provided by Alessia Piazza, PhD, of Excerpta Medica, funded by Sanofi-Genzyme and Regeneron Pharmaceuticals Inc.

\section{Funding}

This work was supported by Sanofi and Regeneron Pharmaceuticals, Inc.

\section{Disclosure}

IDP has received speakers' honoraria from Aerocrine, Almirall, AstraZeneca, Boehringer Ingelheim, GSK, Novartis, Teva; fees for organization of educational events from AstraZeneca, Teva; has been on advisory boards of Almirall, AstraZeneca, Boehringer Ingelheim, Chiesi,
Circassia, Dey, Genentech, GSK, Knopp, Merck, MSD, Napp, Novartis, Regeneron Pharmaceuticals, Inc., Respivert, Sanofi, Schering-Plough, Teva; has received traveling grants from AstraZeneca, Boehringer Ingelheim, Chiesi, GSK, Napp, Teva; has received clinical trial support from Chiesi. SS has provided advisory services/participated in advisory boards for AstraZeneca, Boehringer Ingelheim, GSK, Mundipharma, Napp, Novartis, Owlstone Medical; has received speaker fees from Chiesi, ERT Medical, Novartis, Roche, European Respiratory Society, all outside the submitted work. AP has received grants, personal fees, and nonfinancial support from AstraZeneca, Boehringer Ingelheim, Chiesi, GSK, Mundipharma, Roche, Edmond Pharma, Fondazione Maugeri, Fondazione Chiesi, Teva; has received personal fees, nonfinancial support from Menarini, Novartis, Zambon; has received grants from Sanofi (all outside the submitted work). JC has received research support from Sanofi, AstraZeneca, Genentech, Novartis, Regeneron, Sanofi, Optinose. LDS has been advisor for Aimmune, Optinose, Regeneron Pharmaceuticals, Inc., Sanofi Genzyme; has received speaker fees from Regeneron Pharmaceuticals, Inc., Sanofi Genzyme; has received clinical trials funding from Aimmune, Amgen, AstraZeneca, Circassia, DBV, Galderma, GSK, Lupin, Merck, Mylan, Novartis, Novo Nordisk, Optinose, Pearl, Pfizer, Pulmagen, Roxane, Sanofi, Spirometrix, Teva, Vectura, Watson. PB has received consultant and speaker fees from AstraZeneca, Boehringer Ingelheim, GSK, Novartis, Teva. DL has received research funding from Sanofi. MSR, PR, HWS, NP, AT are employees, and may hold stock and/or stock options in Sanofi. YD, MR, NMHG are employees and shareholders of Regeneron Pharmaceuticals, Inc. H-SP has no conflicts of interest to disclose. The authors report no other conflicts of interest in this work.

\section{References}

1. Global Initiative for Asthma (GINA). Global strategy for asthma management and prevention; 2018. Available from: https:// ginasthma.org/wp-content/uploads/2018/04/wms-GINA-2018-reporttracked_v1.3.pdf. Accessed August 4, 2020.

2. Flood-Page P, Swenson C, Faiferman I, et al. A study to evaluate safety and efficacy of mepolizumab in patients with moderate persistent asthma. Am J Respir Crit Care Med. 2007;176(11):1062-1071. doi:10.1164/rccm.200701-085OC

3. Kips JC, O'Connor BJ, Langley SJ, et al. Effect of SCH55700, a humanized anti-human interleukin-5 antibody, in severe persistent asthma: a pilot study. Am J Respir Crit Care Med. 2003;167 (12):1655-1659. doi:10.1164/rccm.200206-525OC 
4. Hanania NA, Wenzel S, Rosén K, et al. Exploring the effects of omalizumab in allergic asthma: an analysis of biomarkers in the EXTRA study. Am J Respir Crit Care Med. 2013;187(8):804-811. doi:10.1164/rccm.201208-1414OC

5. MacDonald LE, Karow M, Stevens S, et al. Precise and in situ genetic humanization of $6 \mathrm{Mb}$ of mouse immunoglobulin genes. Proc Natl Acad Sci. 2014;111(14):5147-5152. doi:10.1073/pnas.132 3896111

6. Murphy AJ, Macdonald LE, Stevens S, et al. Mice with megabase humanization of their immunoglobulin genes generate antibodies as efficiently as normal mice. Proc Natl Acad Sci U S A. 2014;111 (14):5153-5158. doi:10.1073/pnas.1324022111

7. Le Floc'h A, Allinne J, Nagashima K, et al. Dual blockade of IL-4 and IL-13 with dupilumab, an IL-4R $\alpha$ antibody, is required to broadly inhibit type 2 inflammation. Allergy. 2020;75(5):1188-1204. doi:10.1111/all.14151

8. Gandhi NA, Pirozzi G, Graham NMH. Commonality of the IL-4/IL13 pathway in atopic diseases. Expert Rev Clin Immunol. 2017;13 (5):425-437. doi:10.1080/1744666X.2017.1298443

9. DUPIXENT ${ }^{\circledR}$ (dupilumab). Highlights of prescribing information. US Food and Drug Administration; 2019. Available from: https:// www.accessdata.fda.gov/drugsatfda_docs/label/2019/761055s014lbl. pdf. Accessed August 4, 2020.

10. DUPIXENT ${ }^{\circledR}$ (dupilumab). Summary of product characteristics. European Medicines Agency; 2017. Available from: http://ec. europa.eu/health/documents/community-register/2019/ 20190506144541/anx_144541_en.pdf. Accessed August 4, 2020.

11. Castro M, Corren J, Pavord ID, et al. Dupilumab efficacy and safety in moderate-to-severe uncontrolled asthma. $N$ Engl J Med. 2018;378 (26):2486-2496. doi:10.1056/NEJMoa1804092
12. Juniper EF, Svensson K, Mork AC, Stahl E. Measurement properties and interpretation of three shortened versions of the asthma control questionnaire. Respir Med. 2005;99(5):553-558. doi:10.1016/j. rmed.2004.10.008

13. Juniper EF, Guyatt GH, Willan A, Griffith LE. Determining a minimal important change in a disease-specific quality of life questionnaire. J Clin Epidemiol. 1994;47(1):81-87. doi:10.1016/ 0895-4356(94)90036-1

14. Castro M, Zangrilli J, Wechsler ME, et al. Reslizumab for inadequately controlled asthma with elevated blood eosinophil counts: results from two multicentre, parallel, double-blind, randomised, placebo-controlled, phase 3 trials. Lancet Respir Med. 2015;3 (5):355-366. doi:10.1016/S2213-2600(15)00042-9

15. Rabe KF, Nair P, Brusselle G, et al. Efficacy and safety of dupilumab in glucocorticoid-dependent severe asthma. N Engl J Med. 2018;378 (26):2475-2485. doi:10.1056/NEJMoa1804093

16. Wenzel S, Castro M, Corren J, et al. Dupilumab efficacy and safety in adults with uncontrolled persistent asthma despite use of medium-tohigh-dose inhaled corticosteroids plus a long-acting $\beta 2$ agonist: a randomised double-blind placebo-controlled pivotal phase $2 \mathrm{~b}$ doseranging trial. Lancet. 2016;388(10039):31-44. doi:10.1016/S01406736(16)30307-5

17. Gandhi NA, Bennett BL, Graham NM, Pirozzi G, Stahl N, Yancopoulos GD. Targeting key proximal drivers of type 2 inflammation in disease. Nat Rev Drug Discov. 2016;15:35-50. doi:10. $1038 / \mathrm{nrd} 4624$

18. Locksley RM. Asthma and allergic inflammation. Cell. 2010;140 (6):777-783. doi:10.1016/j.cell.2010.03.004

\section{Publish your work in this journal}

The Journal of Asthma and Allergy is an international, peer-reviewed open-access journal publishing original research, reports, editorials and commentaries on the following topics: Asthma; Pulmonary physiology; Asthma related clinical health; Clinical immunology and the immunological basis of disease; Pharmacological interventions and new therapies. The manuscript management system is completely online and includes a very quick and fair peer-review system, which is all easy to use. Visit http://www.dovepress.com/testimonials.php to read real quotes from published authors. 